\title{
SOME ASPECTS OF GROWTH WITH NORMS FROM BIRTH TO 18 YEARS
}

BY

\author{
W. H. HAMMOND \\ Ministry of Health, London
}

Several cross-sectional studies of children's body measurements have been made from which average growth may be inferred (Hammond, 1953a; Provis and Ellis, 1955; Clements and Pickett, 1955 and 1956 for school ages. Low, 1952; Acheson and Hewitt, 1954; Hammond, 1955; Thomson, 1956 for pre-school ages). But it is impossible to assess the significance of departures from average growth unless normal growth variability is known, and this can be ascertained only from actual differences shown by individuals between successive measuring occasions.

Usually growth is studied over one year, but its consistency over longer periods can be assessed by comparing successive years' growth with each other and with that of the longer periods. The data used in this paper, collected from a number of different studies, are based on growth measurements taken over intervals of 1 to 3 years from birth to late adolescence. They consist of measurements of growth taken by the author in the following investigations:

(1) A pilot survey of infants' growth from birth to 5 years (Hammond, 1955);

(2) A Ministry of Health survey of the growth and development of children of different socioeconomic levels aged 5 to 18 years (Hammond, 1953b);

(3) Growth records taken at Camp Schools (unpublished Ph.D. thesis; University of London);

(4) A growth survey at the National Children's Home, Harpenden (unpublished);

(5) A nutritional survey at Rotherham (in the press).

\section{MEASURING ERrors}

Growth represents the difference between two measurements and it is therefore subject to the ordinary errors of measurement on either occasion; hence standard deviations of errors of growth tend to be $\sqrt{ } 2$ times those of single measurements. Constant errors (e.g. in instruments or technique) which apply to both measuring occasions, leave the mean growth and its variability unaffected. Random errors increase the variability of growth rather than its mean but, more important, they affect two adjacent years' growth in opposite ways, tending to produce apparent inconsistency in growth. As the total coefficients of variation of growth are high and the error variance seldom exceeds 5 per cent. of the growth variance for any year, no allowance for errors has been made either to the means or to the correlations reported later.

\section{Age and Growth Patterns}

For the purpose of providing growth norms, children considered to be suffering from clinical abnormality or malnutrition have been excluded from Tables I and II (overleaf) and Figs 1 and 2 (overleaf). For both weight and height, the annual increment is greatest in the first year of life. Thereafter the weight increment remains fairly constant until about the fifth year and then gradually increases until the pubertal spurt is reached. The annual height increment, on the other hand, decreases, until the fifth year and then remains fairly constant until the pubertal spurt. This pattern, of course, is fairly well known from cross-sectional studies.

It is perhaps not generally realized that, on average, weight and height growth during the first year of life are-even in absolute amounts-greater than during any other year. When expressed as a proportion of the attained measurements, growth during the first years is relatively greater, and the pattern is then one of progressive decrease, arrested temporarily by the pubertal spurt.

Development occurs at different rates in individuals, and growth should be compared with the 
TABLE I

WEIGHT GROWTH OVER 1, 2, AND 3 YEARS, BY SEX

\begin{tabular}{|c|c|c|c|c|c|c|c|c|c|c|}
\hline \multirow{2}{*}{$\begin{array}{l}\text { Ini- } \\
\text { tial } \\
\text { Age } \\
\text { (yrs) }\end{array}$} & \multirow[b]{2}{*}{ Sex } & \multicolumn{3}{|c|}{1 Year } & \multicolumn{3}{|c|}{2 Years } & \multicolumn{3}{|c|}{3 Years } \\
\hline & & No. & $\begin{array}{c}\text { Mean } \\
\text { (kg.) }\end{array}$ & s.d. & No. & $\begin{array}{c}\text { Mean } \\
(\mathrm{kg} .)\end{array}$ & s.d. & No. & $\begin{array}{c}\text { Mean } \\
(\mathrm{kg} .)\end{array}$ & s.d. \\
\hline Birth & $\begin{array}{l}\text { Boys } \\
\text { Girls }\end{array}$ & $\begin{array}{l}214 \\
237 \\
\end{array}$ & \begin{tabular}{l|}
$6 \cdot 89$ \\
6.62 \\
\end{tabular} & \begin{tabular}{|l|}
$1 \cdot 00$ \\
$1 \cdot 27$ \\
\end{tabular} & $\begin{array}{l}326 \\
290\end{array}$ & \begin{tabular}{r|}
$9 \cdot 71$ \\
$10 \cdot 70$ \\
\end{tabular} & \begin{tabular}{|l|}
$1 \cdot 77$ \\
$1 \cdot 22$ \\
\end{tabular} & $\begin{array}{l}204 \\
205 \\
\end{array}$ & $\begin{array}{l}11 \cdot 38 \\
12 \cdot 29 \\
\end{array}$ & $\begin{array}{l}1.68 \\
2 \cdot 00 \\
\end{array}$ \\
\hline 1 & $\begin{array}{l}\text { Boys } \\
\text { Girls }\end{array}$ & $\begin{array}{l}44 \\
45 \\
\end{array}$ & $\begin{array}{l}2.04 \\
2.00 \\
\end{array}$ & $\begin{array}{l}0.54 \\
0.64 \\
\end{array}$ & $\begin{array}{l}34 \\
34 \\
\end{array}$ & $\begin{array}{l}4 \cdot 58 \\
4 \cdot 58 \\
\end{array}$ & \begin{tabular}{|l|}
0.73 \\
0.73 \\
\end{tabular} & $\begin{array}{l}41 \\
41\end{array}$ & $\begin{array}{l}6 \cdot 30 \\
6 \cdot 30 \\
\end{array}$ & $\begin{array}{r}1 \cdot 18 \\
1 \cdot 18 \\
\end{array}$ \\
\hline 2 & $\begin{array}{l}\text { Boys } \\
\text { Girls } \\
\end{array}$ & $\begin{array}{l}24 \\
34 \\
\end{array}$ & $\begin{array}{l}\cdot 72 \\
2 \cdot 18 \\
\end{array}$ & \begin{tabular}{|l|}
0.45 \\
0.54 \\
\end{tabular} & $\begin{array}{l}36 \\
33 \\
\end{array}$ & $\begin{array}{l}3 \cdot 76 \\
4 \cdot 04 \\
\end{array}$ & $\begin{array}{l}0.91 \\
0.64 \\
\end{array}$ & $\begin{array}{l}35 \\
31 \\
\end{array}$ & $\begin{array}{l}5 \cdot 40 \\
6 \cdot 03\end{array}$ & $\begin{array}{l}1 \cdot 27 \\
0.86\end{array}$ \\
\hline 3 & $\begin{array}{l}\text { Boys } \\
\text { Girls } \\
\end{array}$ & $\begin{array}{l}27 \\
37 \\
\end{array}$ & \begin{tabular}{l|}
$2 \cdot 00$ \\
$2 \cdot 09$ \\
\end{tabular} & \begin{tabular}{|l|}
1.04 \\
0.64 \\
\end{tabular} & $\begin{array}{l}19 \\
33 \\
\end{array}$ & $\begin{array}{l}3 \cdot 13 \\
3 \cdot 95 \\
\end{array}$ & $\begin{array}{l}1.68 \\
0.67 \\
\end{array}$ & 二 & 二 & 二 \\
\hline 4 & $\begin{array}{l}\text { Boys } \\
\text { Girls } \\
\end{array}$ & $\begin{array}{l}38 \\
41 \\
\end{array}$ & $\begin{array}{c}1.72 \\
2.00 \\
\end{array}$ & $\begin{array}{l}0.91 \\
0.77\end{array} \mid$ & $\begin{array}{r}7 \\
(2)\end{array}$ & $\begin{array}{l}4 \cdot 35 \\
2 \cdot 86 \\
\end{array}$ & $1 \cdot 36$ & 二 & $=$ & 二 \\
\hline 5 & $\begin{array}{l}\text { Boys } \\
\text { Girls } \\
\end{array}$ & $\begin{array}{r}59 \\
46 \\
\end{array}$ & $\begin{array}{l}2 \cdot 04 \\
2 \cdot 14 \\
\end{array}$ & $\begin{array}{l}1.00 \\
0.86 \\
\end{array}$ & $\begin{array}{r}6 \\
11 \\
\end{array}$ & $\begin{array}{r}4 \cdot 35 \\
4.94 \\
\end{array}$ & $\begin{array}{l}1.86 \\
0.91 \\
\end{array}$ & $\begin{array}{l}29 \\
46 \\
\end{array}$ & $\begin{array}{l}6 \cdot 03 \\
6 \cdot 49 \\
\end{array}$ & \begin{tabular}{|l|}
1.68 \\
1.32 \\
\end{tabular} \\
\hline 6 & $\begin{array}{l}\text { Boys } \\
\text { Girls }\end{array}$ & $\begin{array}{l}51 \\
55 \\
\end{array}$ & $\begin{array}{l}2 \cdot 18 \\
2 \cdot 49 \\
\end{array}$ & $\begin{array}{l}1 \cdot 04 \\
0.86 \\
\end{array}$ & $\begin{array}{l}(3) \\
15 \\
\end{array}$ & $\begin{array}{l}4 \cdot 72 \\
4 \cdot 72 \\
\end{array}$ & $\overline{0.77}$ & $\begin{array}{l}34 \\
41 \\
\end{array}$ & $\begin{array}{l}6 \cdot 94 \\
6 \cdot 21 \\
\end{array}$ & $\begin{array}{r}2 \cdot 18 \\
1 \cdot 54 \\
\end{array}$ \\
\hline 7 & $\begin{array}{l}\text { Boys } \\
\text { Girls }\end{array}$ & $\begin{array}{l}57 \\
69\end{array}$ & $\begin{array}{l}2 \cdot 31 \\
2 \cdot 45\end{array}$ & \begin{tabular}{|l|}
0.82 \\
1.19 \\
\end{tabular} & $\begin{array}{r}7 \\
28 \\
\end{array}$ & $\begin{array}{l}4 \cdot 35 \\
5 \cdot 49\end{array}$ & $\begin{array}{l}0.96 \\
1.59 \\
\end{array}$ & $\begin{array}{l}35 \\
55\end{array}$ & $\begin{array}{l}7 \cdot 03 \\
7 \cdot 48 \\
\end{array}$ & \begin{tabular}{|l|}
$1 \cdot 68$ \\
$2 \cdot 40$ \\
\end{tabular} \\
\hline 8 & $\begin{array}{l}\text { Boys } \\
\text { Girls } \\
\end{array}$ & $\begin{array}{l}79 \\
96 \\
\end{array}$ & $\begin{array}{l}2 \cdot 54 \\
2.86 \\
\end{array}$ & \begin{tabular}{|l|}
$1 \cdot 27$ \\
$1 \cdot 27$ \\
\end{tabular} & $\begin{array}{l}20 \\
30\end{array}$ & $\begin{array}{l}5 \cdot 81 \\
7 \cdot 48 \\
\end{array}$ & $\begin{array}{r}1 \cdot 90 \\
3 \cdot 72 \\
\end{array}$ & $\begin{array}{l}62 \\
84 \\
\end{array}$ & $\begin{array}{l}8 \cdot 48 \\
7 \cdot 71 \\
\end{array}$ & \begin{tabular}{|l|}
$2 \cdot 13$ \\
$3 \cdot 36$ \\
\end{tabular} \\
\hline 9 & $\begin{array}{l}\text { Boys } \\
\text { Girls } \\
\end{array}$ & $\begin{array}{r}109 \\
93 \\
\end{array}$ & $\begin{array}{l}2 \cdot 63 \\
2 \cdot 81 \\
\end{array}$ & $\begin{array}{r}1.09 \\
1.40 \\
\end{array}$ & $\begin{array}{l}42 \\
21 \\
\end{array}$ & $\begin{array}{l}6.03 \\
7.85 \\
\end{array}$ & $\begin{array}{l}2 \cdot 13 \\
4 \cdot 17 \\
\end{array}$ & $\begin{array}{l}95 \\
70 \\
\end{array}$ & $\begin{array}{l}9 \cdot 39 \\
9 \cdot 57 \\
\end{array}$ & \begin{tabular}{|l|}
2.99 \\
3.90 \\
\end{tabular} \\
\hline 10 & $\begin{array}{l}\text { Boys } \\
\text { Girls }\end{array}$ & $\begin{array}{l}99 \\
84 \\
\end{array}$ & $\begin{array}{l}3 \cdot 04 \\
3 \cdot 72 \\
\end{array}$ & $\begin{array}{l}1 \cdot 27 \\
1.73 \\
\end{array}$ & $\begin{array}{l}68 \\
25 \\
\end{array}$ & $\begin{array}{l}7 \cdot 85 \\
9 \cdot 30\end{array}$ & $\begin{array}{l}1 \cdot 95 \\
4 \cdot 22 \\
\end{array}$ & $\begin{array}{l}46 \\
67 \\
\end{array}$ & \begin{tabular}{|l|}
$10 \cdot 34$ \\
$11 \cdot 88$ \\
\end{tabular} & $\begin{array}{r}3 \cdot 45 \\
3 \cdot 76 \\
\end{array}$ \\
\hline 11 & $\begin{array}{l}\text { Boys } \\
\text { Girls } \\
\end{array}$ & $\begin{array}{l}96 \\
88 \\
\end{array}$ & $\begin{array}{l}.49 \\
4.49 \\
\end{array}$ & $\begin{array}{l}1 \cdot 73 \\
2 \cdot 00 \\
\end{array}$ & $\begin{array}{l}28 \\
24 \\
\end{array}$ & $\begin{array}{r}8 \cdot 71 \\
11 \cdot 15 \\
\end{array}$ & \begin{tabular}{|l|}
$2 \cdot 40$ \\
$3 \cdot 45$ \\
\end{tabular} & $\begin{array}{l}34 \\
76 \\
\end{array}$ & \begin{tabular}{|l|}
$13 \cdot 38$ \\
$13 \cdot 74$ \\
\end{tabular} & \begin{tabular}{|l|}
$4 \cdot 67$ \\
$3 \cdot 67$ \\
\end{tabular} \\
\hline 12 & $\begin{array}{l}\text { Boys } \\
\text { Girls } \\
\end{array}$ & $\begin{array}{l}69 \\
80 \\
\end{array}$ & \begin{tabular}{rl|}
$4 \cdot 58$ \\
5.03 \\
\end{tabular} & $\begin{array}{l}2 \cdot 82 \\
1 \cdot 84 \\
\end{array}$ & $\begin{array}{l}19 \\
31 \\
\end{array}$ & \begin{tabular}{ll|}
$10 \cdot 39$ \\
$11 \cdot 43$ \\
\end{tabular} & $\begin{array}{l}2 \cdot 99 \\
2 \cdot 72 \\
\end{array}$ & $\begin{array}{l}33 \\
34 \\
\end{array}$ & $\begin{array}{l}12 \cdot 97 \\
12 \cdot 34 \\
\end{array}$ & $\begin{array}{l}4 \cdot 17 \\
3 \cdot 63 \\
\end{array}$ \\
\hline 13 & $\begin{array}{l}\text { Boys } \\
\text { Girls } \\
\end{array}$ & $\begin{array}{l}41 \\
70 \\
\end{array}$ & \begin{tabular}{|l|}
5.35 \\
4.86 \\
\end{tabular} & $\begin{array}{l}2 \cdot 54 \\
2 \cdot 18 \\
\end{array}$ & $\begin{array}{r}11 \\
6 \\
\end{array}$ & $\begin{array}{r}12 \cdot 24 \\
8 \cdot 21 \\
\end{array}$ & $\begin{array}{l}4 \cdot 35 \\
4 \cdot 13 \\
\end{array}$ & $\begin{array}{l}43 \\
46 \\
\end{array}$ & $\begin{array}{l}13 \cdot 74 \\
10 \cdot 84 \\
\end{array}$ & \begin{tabular}{|l|}
$4 \cdot 76$ \\
$3 \cdot 63$ \\
\end{tabular} \\
\hline 14 & $\begin{array}{l}\text { Boys } \\
\text { Girls } \\
\end{array}$ & $\begin{array}{l}55 \\
66 \\
\end{array}$ & $\begin{array}{l}5 \cdot 81 \\
2 \cdot 86 \\
\end{array}$ & $\begin{array}{l}2 \cdot 40 \\
2 \cdot 00 \\
\end{array}$ & (3) & $\begin{array}{c}8 \cdot 39 \\
- \\
\end{array}$ & - & $\begin{array}{l}44 \\
49 \\
\end{array}$ & $\begin{array}{r}10.44 \\
6.80 \\
\end{array}$ & $\begin{array}{l}5 \cdot 26 \\
4 \cdot 85 \\
\end{array}$ \\
\hline 15 & $\begin{array}{l}\text { Boys } \\
\text { Girls } \\
\end{array}$ & $\begin{array}{l}51 \\
43 \\
\end{array}$ & \begin{tabular}{|l|}
$4 \cdot 63$ \\
$2 \cdot 27$ \\
\end{tabular} & $\begin{array}{r}2.99 \\
1.72 \\
\end{array}$ & (3) & $\begin{array}{c}8 \cdot 35 \\
-\end{array}$ & 二 & 10 & $\begin{array}{c}10 \cdot 25 \\
-\end{array}$ & 3.45 \\
\hline 16 & $\begin{array}{l}\text { Boys } \\
\text { Girls } \\
\end{array}$ & $\begin{array}{l}51 \\
30 \\
\end{array}$ & $\begin{array}{l}3.08 \\
0.27 \\
\end{array}$ & $\begin{array}{l}2 \cdot 31 \\
1 \cdot 22 \\
\end{array}$ & 二 & 二 & $=$ & 二 & 二 & - \\
\hline 17 & $\begin{array}{l}\text { Boys } \\
\text { Girls }\end{array}$ & 28 & $\begin{array}{c}2 \cdot 86 \\
-\end{array}$ & $\begin{array}{c}2 \cdot 09 \\
-\end{array}$ & (2) & $\frac{4 \cdot 54}{-}$ & 二 & 二 & 二 & - \\
\hline
\end{tabular}

appropriate maturity level rather than with the chronologically equivalent age, especially around puberty. At this age the mean growth is affected by the accelerated growth during puberty of the earliest maturer and continues to be affected until the latest maturer has finished his spurt; hence standard deviations are high and the pattern of the means is flattened and may not be followed by any individual. Nevertheless the mean will reflect the proportion who have actually reached their spurt at the different ages. The relation between growth and puberty is sufficiently well defined to indicate whether or not a growth spurt should be expected in any individual. After age 10 the expected growth will be
TABLE II

HEIGHT GROWTH OVER 1, 2, AND 3 YEARS

\begin{tabular}{|c|c|c|c|c|c|c|c|c|c|c|}
\hline \multirow{2}{*}{$\begin{array}{r}\text { Ini- } \\
\text { tial } \\
\text { Age } \\
\text { (yrs) } \\
\end{array}$} & \multirow{2}{*}{ Sex } & \multicolumn{3}{|c|}{1 year } & \multicolumn{3}{|c|}{2 years } & \multicolumn{3}{|c|}{3 years } \\
\hline & & No. & $\begin{array}{l}\text { Mean } \\
(\mathrm{cm} .)\end{array}$ & s.d. & No. & $\begin{array}{l}\text { Mean } \\
(\mathrm{cm} .)\end{array}$ & s.d. & No. & $\begin{array}{l}\text { Mean } \\
(\mathrm{cm} .)\end{array}$ & s.d. \\
\hline Birth & $\begin{array}{l}\text { Boys } \\
\text { Girls } \\
\end{array}$ & $\begin{array}{l}214 \\
237 \\
\end{array}$ & $\begin{array}{l}24 \cdot 8 \\
24 \cdot 2 \\
\end{array}$ & $\begin{array}{l}2 \cdot 5 \\
2 \cdot 4 \\
\end{array}$ & $\begin{array}{l}42 \\
37 \\
\end{array}$ & $\begin{array}{l}33 \cdot 3 \\
33 \cdot 6 \\
\end{array}$ & $\begin{array}{l}3 \cdot 8 \\
2 \cdot 1 \\
\end{array}$ & $\begin{array}{l}23 \\
32 \\
\end{array}$ & $\begin{array}{r}42 \cdot 6 \\
43 \cdot 9 \\
\end{array}$ & $\begin{array}{l}3 \cdot 9 \\
2 \cdot 6 \\
\end{array}$ \\
\hline 1 & $\begin{array}{l}\text { Boys } \\
\text { Girls } \\
\end{array}$ & $\begin{array}{l}44 \\
45 \\
\end{array}$ & $\begin{array}{l}11.0 \\
11.7 \\
\end{array}$ & $\begin{array}{l}1.7 \\
1.8 \\
\end{array}$ & $\begin{array}{l}24 \\
35 \\
\end{array}$ & $\begin{array}{l}22 \cdot 7 \\
22 \cdot 1 \\
\end{array}$ & $\begin{array}{l}2 \cdot 3 \\
2 \cdot 5 \\
\end{array}$ & $\begin{array}{l}34 \\
41 \\
\end{array}$ & $\begin{array}{r}29 \cdot 5 \\
30 \cdot 5 \\
\end{array}$ & $\begin{array}{l}2 \cdot 3 \\
2 \cdot 7 \\
\end{array}$ \\
\hline 2 & $\begin{array}{l}\text { Boys } \\
\text { Girls } \\
\end{array}$ & $\begin{array}{l}23 \\
34 \\
\end{array}$ & $\begin{array}{r}9 \cdot 3 \\
10 \cdot 3 \\
\end{array}$ & $\begin{array}{l}1 \cdot 3 \\
0.8 \\
\end{array}$ & $\begin{array}{l}33 \\
34 \\
\end{array}$ & $\begin{array}{l}15 \cdot 8 \\
16 \cdot 0 \\
\end{array}$ & $\begin{array}{l}1.3 \\
1.6 \\
\end{array}$ & \begin{tabular}{|l|}
37 \\
32 \\
\end{tabular} & $\begin{array}{r}22 \cdot 1 \\
22 \cdot 2 \\
\end{array}$ & $\begin{array}{l}1 \cdot 9 \\
2 \cdot 2 \\
\end{array}$ \\
\hline 3 & $\begin{array}{l}\text { Boys } \\
\text { Girls } \\
\end{array}$ & $\begin{array}{l}21 \\
31 \\
\end{array}$ & $\begin{array}{l}7 \cdot 4 \\
7 \cdot 6\end{array}$ & $\begin{array}{l}1 \cdot 3 \\
1 \cdot 2\end{array}$ & $\begin{array}{l}23 \\
33\end{array}$ & $\begin{array}{r}13.3 \\
13.7 \\
\end{array}$ & $\begin{array}{r}1.9 \\
1.4 \\
\end{array}$ & $=$ & - & $=$ \\
\hline 4 & $\begin{array}{l}\text { Boys } \\
\text { Girls } \\
\end{array}$ & $\begin{array}{l}24 \\
26 \\
\end{array}$ & $\begin{array}{l}3 \cdot 7 \\
4 \cdot 3 \\
\end{array}$ & $\begin{array}{l}1.9 \\
0.9 \\
\end{array}$ & $=$ & - & - & 二 & - & - \\
\hline 5 & $\begin{array}{l}\text { Boys } \\
\text { Girls } \\
\end{array}$ & $\begin{array}{l}45 \\
45 \\
\end{array}$ & $\begin{array}{l}6 \cdot 1 \\
5 \cdot 6 \\
\end{array}$ & \begin{tabular}{|l|}
$1 \cdot 3$ \\
$1 \cdot 1$ \\
\end{tabular} & $\overline{7}$ & $1 \overline{11 \cdot 6}$ & $\overline{1 \cdot 1}$ & $\begin{array}{l}29 \\
39 \\
\end{array}$ & \begin{tabular}{l|}
17.9 \\
16.4 \\
\end{tabular} & $\begin{array}{l}2 \cdot 3 \\
2 \cdot 7 \\
\end{array}$ \\
\hline 6 & $\begin{array}{l}\text { Boys } \\
\text { Girls } \\
\end{array}$ & $\begin{array}{l}42 \\
55 \\
\end{array}$ & $\begin{array}{l}5 \cdot 3 \\
5 \cdot 3 \\
\end{array}$ & $\begin{array}{l}1 \cdot 3 \\
1.5 \\
\end{array}$ & $\overline{9}$ & $\overline{11 \cdot 2}$ & $\overline{1.6}$ & $\begin{array}{l}34 \\
32 \\
\end{array}$ & $\begin{array}{l}17 \cdot 0 \\
16 \cdot 4 \\
\end{array}$ & \begin{tabular}{|l}
2.4 \\
1.8 \\
\end{tabular} \\
\hline 7 & $\begin{array}{l}\text { Boys } \\
\text { Girls }\end{array}$ & $\begin{array}{l}71 \\
72\end{array}$ & $\begin{array}{l}5 \cdot 7 \\
4 \cdot 6\end{array}$ & $\begin{array}{l}1.0 \\
1.0\end{array}$ & $\begin{array}{l}7 \\
6\end{array}$ & $\begin{array}{l}10 \cdot 7 \\
11 \cdot 2\end{array}$ & $\begin{array}{l}1 \cdot 4 \\
2 \cdot 5 \\
\end{array}$ & \begin{tabular}{|l|}
41 \\
39
\end{tabular} & $\begin{array}{l}15 \cdot 7 \\
15 \cdot 7 \\
\end{array}$ & \begin{tabular}{|l|}
$\cdot 8$ \\
$2 \cdot 3$ \\
\end{tabular} \\
\hline 8 & $\begin{array}{l}\text { Boys } \\
\text { Girls } \\
\end{array}$ & $\begin{array}{l}100 \\
116 \\
\end{array}$ & $\begin{array}{l}5 \cdot 0 \\
4 \cdot 7 \\
\end{array}$ & $\begin{array}{l}0.9 \\
1.0 \\
\end{array}$ & $\begin{array}{l}22 \\
19 \\
\end{array}$ & $\begin{array}{l}10 \cdot 2 \\
11 \cdot 8 \\
\end{array}$ & $\begin{array}{l}1.6 \\
2.8 \\
\end{array}$ & $\begin{array}{l}62 \\
43 \\
\end{array}$ & \begin{tabular}{|l|}
$15 \cdot 0$ \\
$16 \cdot 2$ \\
\end{tabular} & $\begin{array}{l}2 \cdot 0 \\
2 \cdot 0 \\
\end{array}$ \\
\hline 9 & $\begin{array}{l}\text { Boys } \\
\text { Girls }\end{array}$ & $\begin{array}{l}144 \\
125 \\
\end{array}$ & $\begin{array}{l}4 \cdot 6 \\
4 \cdot 8\end{array}$ & $\begin{array}{l}1.2 \\
1.4\end{array}$ & $\begin{array}{l}19 \\
21\end{array}$ & $\begin{array}{r}9 \cdot 1 \\
12 \cdot 2 \\
\end{array}$ & $\begin{array}{l}2 \cdot 2 \\
2 \cdot 4 \\
\end{array}$ & $\begin{array}{l}93 \\
40 \\
\end{array}$ & $\begin{array}{l}14.4 \\
16.4 \\
\end{array}$ & $\begin{array}{l}2 \cdot 2 \\
3 \cdot 4 \\
\end{array}$ \\
\hline 10 & $\begin{array}{l}\text { Boys } \\
\text { Girls }\end{array}$ & $\begin{array}{l}123 \\
123 \\
\end{array}$ & $\begin{array}{l}4 \cdot 5 \\
5 \cdot 3 \\
\end{array}$ & $\begin{array}{l}1 \cdot 1 \\
2 \cdot 1 \\
\end{array}$ & $\begin{array}{l}16 \\
53 \\
\end{array}$ & $\begin{array}{l}10 \cdot 6 \\
13 \cdot 8 \\
\end{array}$ & $\begin{array}{l}2.9 \\
2.9 \\
\end{array}$ & $\begin{array}{l}37 \\
42 \\
\end{array}$ & $\begin{array}{l}14 \cdot 9 \\
18 \cdot 2 \\
\end{array}$ & $\begin{array}{l}2 \cdot 9 \\
3 \cdot 6 \\
\end{array}$ \\
\hline 11 & $\begin{array}{l}\text { Boys } \\
\text { Girls } \\
\end{array}$ & $\begin{array}{l}118 \\
124 \\
\end{array}$ & $\begin{array}{l}5 \cdot 0 \\
5 \cdot 8 \\
\end{array}$ & $\begin{array}{l}1 \cdot 8 \\
2 \cdot 2 \\
\end{array}$ & $\begin{array}{l}14 \\
58 \\
\end{array}$ & $\begin{array}{l}12 \cdot 3 \\
13 \cdot 2 \\
\end{array}$ & $\begin{array}{l}3 \cdot 2 \\
2 \cdot 5 \\
\end{array}$ & $\begin{array}{l}27 \\
54 \\
\end{array}$ & $\begin{array}{l}17.3 \\
15.6 \\
\end{array}$ & $\begin{array}{r}4 \cdot 0 \\
3 \cdot 6 \\
\end{array}$ \\
\hline 12 & $\begin{array}{l}\text { Boys } \\
\text { Girls } \\
\end{array}$ & $\begin{array}{r}80 \\
117 \\
\end{array}$ & $\begin{array}{l}6 \cdot 8 \\
5 \cdot 4 \\
\end{array}$ & $\begin{array}{l}2 \cdot 4 \\
2 \cdot 0 \\
\end{array}$ & $\begin{array}{l}17 \\
56 \\
\end{array}$ & $\begin{array}{l}12 \cdot 5 \\
10 \cdot 8 \\
\end{array}$ & $\begin{array}{l}3 \cdot 2 \\
2 \cdot 7 \\
\end{array}$ & $\begin{array}{l}18 \\
(3) \\
\end{array}$ & $\begin{array}{l}18.6 \\
14.7 \\
\end{array}$ & 4.3 \\
\hline 13 & $\begin{array}{l}\text { Boys } \\
\text { Girls } \\
\end{array}$ & $\begin{array}{l}70 \\
96 \\
\end{array}$ & $\begin{array}{l}6 \cdot 6 \\
4 \cdot 1 \\
\end{array}$ & \begin{tabular}{|l|}
$2 \cdot 5$ \\
1.8 \\
\end{tabular} & $\begin{array}{r}9 \\
51 \\
\end{array}$ & $\begin{array}{r}13.7 \\
6.9 \\
\end{array}$ & $\begin{array}{l}2.9 \\
3.9 \\
\end{array}$ & (1) & $\begin{array}{c}17 \cdot 0 \\
-\end{array}$ & $=$ \\
\hline 14 & $\begin{array}{l}\text { Boys } \\
\text { Girls } \\
\end{array}$ & $\begin{array}{l}69 \\
65 \\
\end{array}$ & $\begin{array}{l}5 \cdot 8 \\
2 \cdot 1 \\
\end{array}$ & $\begin{array}{l}2 \cdot 3 \\
1 \cdot 3 \\
\end{array}$ & $\begin{array}{l}46 \\
50 \\
\end{array}$ & $\begin{array}{r}12.9 \\
3.9 \\
\end{array}$ & \begin{tabular}{|l|}
$4 \cdot 0$ \\
$3 \cdot 3$ \\
\end{tabular} & 二 & 二 & $=$ \\
\hline 15 & $\begin{array}{l}\text { Boys } \\
\text { Girls } \\
\end{array}$ & $\begin{array}{l}46 \\
45 \\
\end{array}$ & $\begin{array}{l}5.0 \\
1.6 \\
\end{array}$ & \begin{tabular}{|l|}
$3 \cdot 0$ \\
$1 \cdot 2$ \\
\end{tabular} & 二 & - & 二 & 二 & - & 二- \\
\hline 16 & $\begin{array}{l}\text { Boys } \\
\text { Girls } \\
\end{array}$ & $\begin{array}{l}44 \\
31 \\
\end{array}$ & $\begin{array}{l}2 \cdot 3 \\
0 \cdot 4 \\
\end{array}$ & $\begin{array}{l}1.9 \\
0.6 \\
\end{array}$ & 二 & 二 & - & 二 & - & - \\
\hline 17 & $\begin{array}{l}\text { Boys } \\
\text { Girls }\end{array}$ & 24 & $1 \cdot 8$ & $\frac{1 \cdot 7}{-}$ & - & $\overline{-}$ & 二 & 二 & 二 & 二 \\
\hline
\end{tabular}

nearer $3 \mathrm{~kg}$. and $4-5 \mathrm{~cm}$. per year for pre-pubescent children and 6-7 kg. and 8-10 $\mathrm{cm}$. per year for children showing physical signs of puberty. Similarly, children maturing early who have already passed their peak spurt will be expected to gain an amount more nearly equal to the post-pubescent growth levels of $2 \cdot 2 \mathrm{~kg}$. and $2 \cdot 5 \mathrm{~cm}$. or less. These figures are intended only for guidance where the stage of puberty is not known with certainty, e.g. as in school records.

Growth over a 2- or 3-year interval will include children who are passing through the various stages of puberty and the effect is to spread the peak growth still further. 


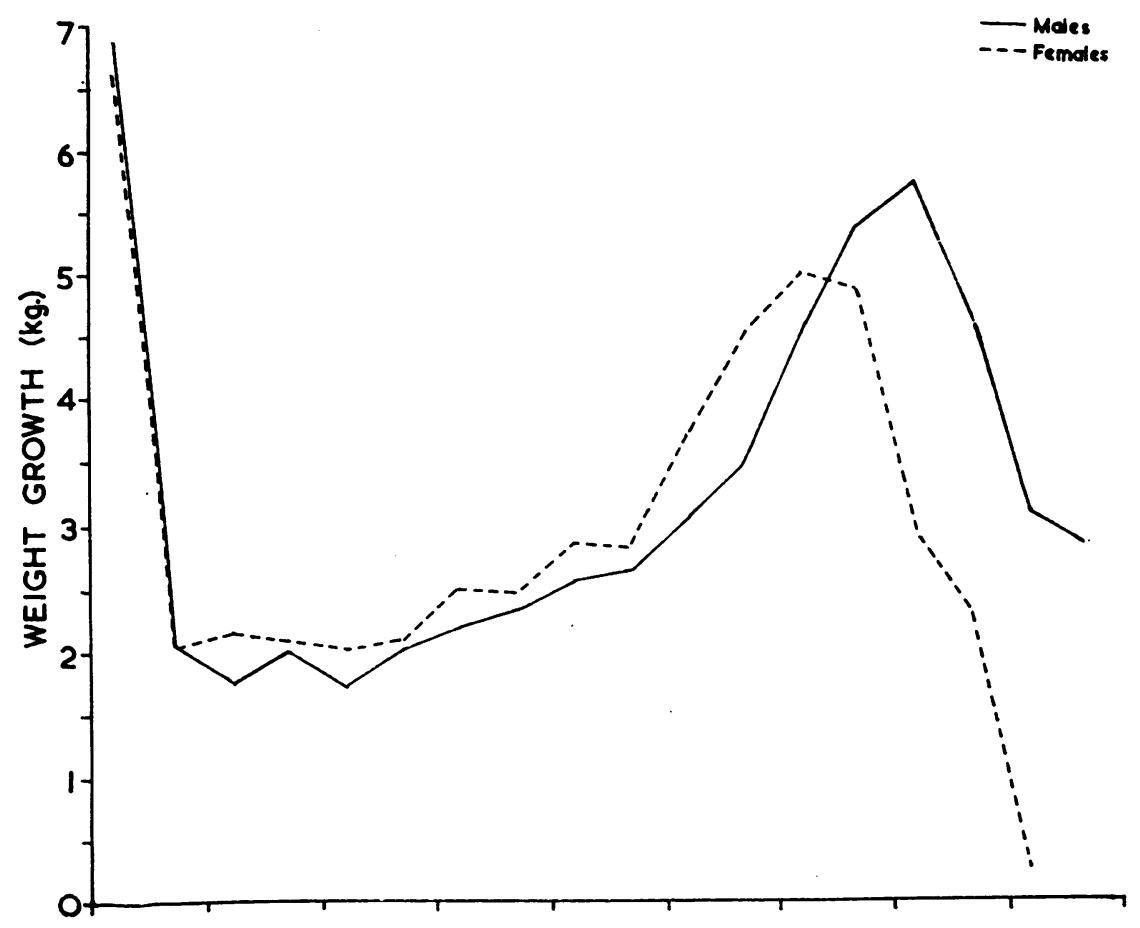

FIG. 1.-Weight growth from birth to 18 years in boys and girls.

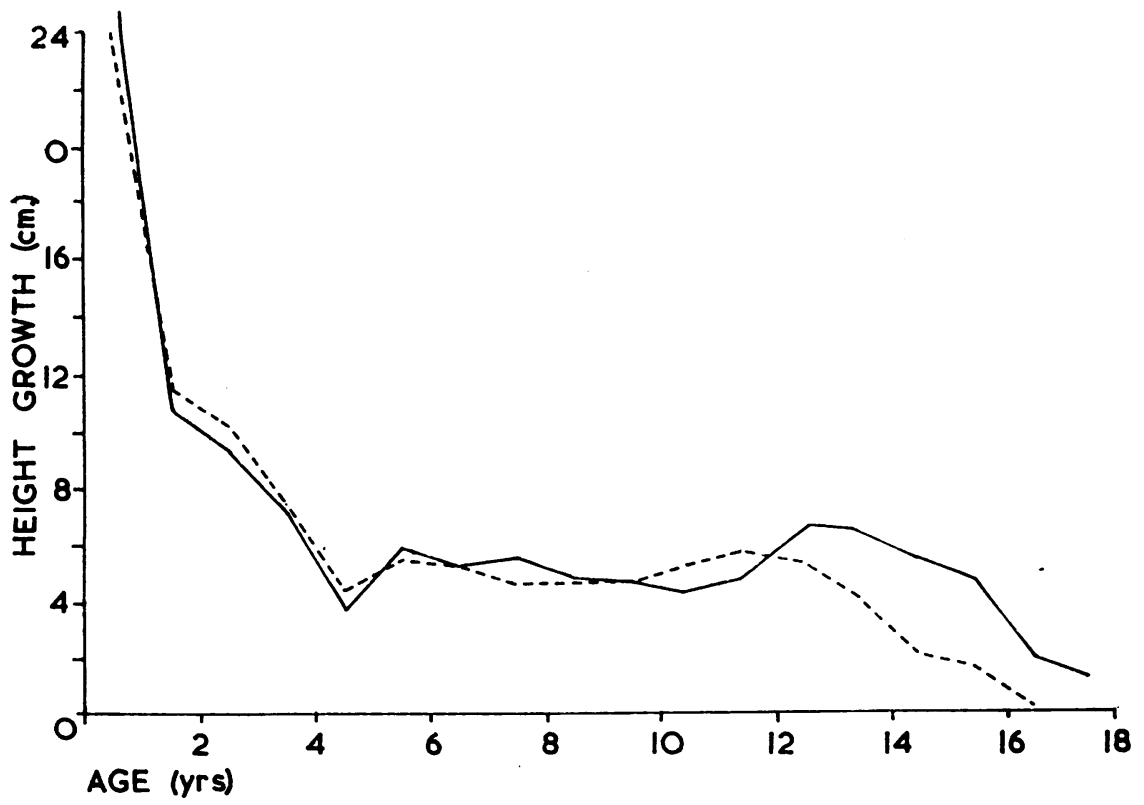

Fig. 2.-Height growth from birth to 18 years in boys and girls. 


\section{Age and Growth Variability}

The variability of weight growth follows a somewhat different pattern from that of the average growth with age, especially during infancy. For example, the average yearly weight growth decreases from approximately six times its standard deviation during the first year to twice the standard deviation during the pubertal years and little more than its standard deviation thereafter. Height growth is less variable and decreases from about seven times its standard deviation up to age 3 to three times its standard deviation around puberty.

The standard deviations of growth in weight and height for two-year intervals are higher for boys than for girls up to ages 5 or 6 . From then until age 11 or 12 , the growth of girls is more variable. The sex difference in variability is due to the different times at which maximum growth occurs in boys and girls. Three-year growth increases maintain a more constant level relative to their variability at different ages. (In this respect weight growth over three years is about comparable with height growth over one year.)

\section{Socio-Economic Class}

The socio-economic status of the child was assessed from the parents' circumstances (occupation and way of living) in Investigations 1, 3, and 5, and as a group characteristic (type of school) in Investigation 2.

The mean weight and height growth of two extreme social groups (mainly professional or executive groups at fee-paying schools compared with working-class children living in slum districts) are shown in Table III and in Table IV (opposite).

Weight growth in the better social group (Group A) exceeds that in Social Group B for boys and girls at nearly all ages, and there is a suggestion that, for girls at least, pubertal growth reaches its peak later and continues later in Social Group B. The annual height increase for boys and girls is clearly greater in Group A than in Group B until late puberty.

TABLE III

MEAN YEARLY WEIGHT GAINS IN TWO SOCIAL GROUPS, BY SEX

\begin{tabular}{|c|c|c|c|c|c|c|c|c|c|c|c|c|}
\hline \multirow{3}{*}{ 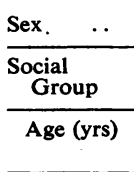 } & \multicolumn{6}{|c|}{ Boys } & \multicolumn{6}{|c|}{ Girls } \\
\hline & \multicolumn{3}{|c|}{$\mathbf{A}$} & \multicolumn{3}{|c|}{ B } & \multicolumn{3}{|c|}{$\mathbf{A}$} & \multicolumn{3}{|c|}{ B } \\
\hline & No. & $\begin{array}{c}\text { Mean } \\
\text { (kg.) }\end{array}$ & s.d. & No. & $\begin{array}{c}\text { Mean } \\
\text { (kg.) }\end{array}$ & s.d. & No. & $\underset{\text { (kg.) }}{\text { Mean }}$ & s.d. & No. & $\begin{array}{l}\text { Mean } \\
\text { (kg.) }\end{array}$ & s.d. \\
\hline $0-1$ & 19 & $7 \cdot 26$ & $1 \cdot 37$ & 195 & 6.99 & $1 \cdot 00$ & 26 & $6 \cdot 49$ & 1.45 & 209 & 6.62 & 1.00 \\
\hline $1-2$ & - & - & - & 44 & $2 \cdot 04$ & 0.54 & - & - & - & 45 & $2 \cdot 00$ & 0.64 \\
\hline $2-3$ & - & - & - & 24 & $1 \cdot 72$ & 0.45 & - & - & - & 34 & $2 \cdot 18$ & 0.54 \\
\hline $3-4$ & - & - & - & 27 & $2 \cdot 00$ & $1 \cdot 04$ & - & - & - & 37 & $2 \cdot 09$ & 0.63 \\
\hline $4-5$ & - & - & - & 36 & 1.68 & 0.86 & - & - & - & 41 & $1 \cdot 72$ & 0.91 \\
\hline $5-6$ & 10 & 1.68 & 1.00 & 49 & $2 \cdot 27$ & 1.00 & 9 & $2 \cdot 54$ & 0.82 & 41 & 1.86 & $0 \cdot 86$ \\
\hline $6-7$ & 7 & $(2 \cdot 1)$ & $1 \cdot 13$ & 47 & $2 \cdot 18$ & 0.64 & 13 & $2 \cdot 86$ & 0.73 & 45 & 2.09 & 0.91 \\
\hline $7-8$ & 7 & $(2 \cdot 4)$ & 0.54 & 58 & $2 \cdot 31$ & 0.95 & 32 & $2 \cdot 86$ & $1 \cdot 32$ & 43 & $2 \cdot 18$ & 0.73 \\
\hline $8-9$ & 31 & $2 \cdot 54$ & $1 \cdot 27$ & 74 & $2 \cdot 49$ & 1.04 & 48 & $3 \cdot 27$ & 1.09 & 72 & $2 \cdot 40$ & $1 \cdot 27$ \\
\hline $9-10$ & 57 & $2 \cdot 86$ & $1 \cdot 22$ & 91 & $2 \cdot 49$ & $1 \cdot 00$ & 36 & 2.95 & $1 \cdot 41$ & 90 & $2 \cdot 77$ & $1 \cdot 18$ \\
\hline $10-11$ & 64 & $3 \cdot 22$ & $1 \cdot 32$ & 78 & $2 \cdot 86$ & 1.09 & 49 & $4 \cdot 17$ & $1 \cdot 86$ & 75 & $3 \cdot 58$ & 1.90 \\
\hline 11-12 & 73 & $3 \cdot 58$ & 1.95 & 74 & $3 \cdot 40$ & 1.50 & 39 & $4 \cdot 54$ & $1 \cdot 86$ & 98 & $4 \cdot 49$ & $2 \cdot 22$ \\
\hline $12-13$ & 19 & 4.98 & 2.99 & 94 & $4 \cdot 08$ & $2 \cdot 90$ & 40 & $4 \cdot 67$ & $1 \cdot 59$ & 96 & $5 \cdot 17$ & $1 \cdot 72$ \\
\hline 13-14 & 38 & $5 \cdot 44$ & $2 \cdot 59$ & 49 & $5 \cdot 31$ & $2 \cdot 00$ & 67 & $3 \cdot 76$ & 2.09 & 56 & $5 \cdot 22$ & $2 \cdot 18$ \\
\hline $14-15$ & 55 & $5 \cdot 58$ & $2 \cdot 40$ & 22 & $5 \cdot 54$ & 3.45 & 60 & $2 \cdot 63$ & $1 \cdot 77$ & 14 & 3.49 & 2.00 \\
\hline $15-16$ & 53 & $4 \cdot 85$ & 2.95 & 10 & $(3 \cdot 4)$ & $1 \cdot 81$ & 43 & $2 \cdot 27$ & $1 \cdot 72$ & - & - & - \\
\hline $16-17$ & 44 & 2.99 & $2 \cdot 31$ & 7 & $(3 \cdot 7)$ & 1.59 & 31 & 0.27 & 0.77 & - & - & - \\
\hline $17-18$ & 24 & $2 \cdot 86$ & $2 \cdot 22$ & 3 & $(2 \cdot 8)$ & - & - & - & - & - & - & - \\
\hline
\end{tabular}


TABLE IV

MEAN YEARLY HEIGHT GAINS IN TWO SOCIAL GROUPS, BY SEX

\begin{tabular}{|c|c|c|c|c|c|c|c|c|c|c|c|c|}
\hline \multirow{3}{*}{ 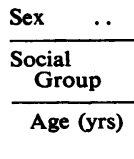 } & \multicolumn{6}{|c|}{ Boys } & \multicolumn{6}{|c|}{ Girls } \\
\hline & \multicolumn{3}{|c|}{$\mathbf{A}$} & \multicolumn{3}{|c|}{ B } & \multicolumn{3}{|c|}{$\mathbf{A}$} & \multicolumn{3}{|c|}{ B } \\
\hline & No. & $\begin{array}{l}\text { Mean } \\
(\mathrm{cm} .)\end{array}$ & s.d. & No. & $\begin{array}{l}\text { Mean } \\
\text { (cm.) }\end{array}$ & s.d. & No. & $\begin{array}{l}\text { Mean } \\
(\mathrm{cm} .)\end{array}$ & s.d. & No. & $\begin{array}{l}\text { Mean } \\
(\mathrm{cm} .)\end{array}$ & s.d. \\
\hline 5 & 10 & $6 \cdot 40$ & - & 33 & $5 \cdot 86$ & $1 \cdot 35$ & 9 & $5 \cdot 61$ & 1.51 & 37 & $5 \cdot 56$ & 1.07 \\
\hline 6 & 7 & $(5 \cdot 47)$ & - & 33 & $5 \cdot 20$ & $1 \cdot 32$ & 13 & $5 \cdot 28$ & 0.90 & 42 & $5 \cdot 30$ & $1 \cdot 54$ \\
\hline 7 & 7 & $(6 \cdot 32)$ & - & 50 & $5 \cdot 11$ & 1.03 & 34 & $5 \cdot 07$ & $1 \cdot 29$ & 41 & $4 \cdot 24$ & $1 \cdot 40$ \\
\hline 8 & 31 & $5 \cdot 22$ & 0.90 & 69 & $4 \cdot 78$ & 0.93 & 45 & $5 \cdot 70$ & $1 \cdot 29$ & 71 & 3.97 & 1.00 \\
\hline 9 & 57 & $5 \cdot 46$ & $1 \cdot 17$ & 97 & $4 \cdot 00$ & 0.82 & 37 & $5 \cdot 50$ & $1 \cdot 27$ & 87 & $4 \cdot 27$ & $1 \cdot 38$ \\
\hline 10 & 64 & $5 \cdot 19$ & $1 \cdot 07$ & 80 & $4 \cdot 20$ & 0.84 & 48 & $6 \cdot 18$ & 1.48 & 73 & $4 \cdot 90$ & $2 \cdot 12$ \\
\hline 11 & 73 & $5 \cdot 42$ & $1 \cdot 76$ & 68 & $4 \cdot 80$ & $1 \cdot 00$ & 37 & $6 \cdot 19$ & $1 \cdot 57$ & 80 & $5 \cdot 55$ & $2 \cdot 20$ \\
\hline 12 & 19 & 5.09 & $2 \cdot 44$ & 82 & $4 \cdot 50$ & $2 \cdot 48$ & 41 & $5 \cdot 05$ & 1.96 & 79 & 5.63 & 1.96 \\
\hline 13 & 38 & $5 \cdot 71$ & $2 \cdot 50$ & 49 & $6 \cdot 24$ & $2 \cdot 42$ & 70 & $3 \cdot 80$ & $1 \cdot 85$ & 30 & $4 \cdot 21$ & 2.02 \\
\hline 14 & 55 & $5 \cdot 60$ & $2 \cdot 32$ & 16 & $7 \cdot 61$ & - & 49 & 1.62 & $1 \cdot 31$ & 5 & $(3 \cdot 1)$ & - \\
\hline 15 & 53 & $4 \cdot 88$ & $2 \cdot 98$ & 2 & $(7 \cdot 6)$ & - & 43 & $1 \cdot 34$ & $1 \cdot 22$ & 2 & $(3 \cdot 0)$ & - \\
\hline 16 & 44 & $2 \cdot 30$ & $1 \cdot 89$ & - & - & - & 30 & 0.37 & 0.59 & - & - & - \\
\hline 17 & 24 & $1 \cdot 83$ & $1 \cdot 75$ & - & - & - & - & - & - & - & - & - \\
\hline
\end{tabular}

\section{Health Status}

Health experience was assessed at the time of measuring.

In the Infant Growth Survey (Investigation 1) the following mean weight gains were made from birth to one year:

\begin{tabular}{l|r|r|r|r|r|r}
\hline \multirow{2}{*}{ Health Experience } & \multicolumn{3}{|c|}{ Boys } & \multicolumn{3}{|c|}{ Girls } \\
\cline { 2 - 6 } & No. & $\begin{array}{c}\text { Mean } \\
(\mathrm{kg} .)\end{array}$ & s.d. & No. & $\begin{array}{c}\text { Mean } \\
\text { (kg.) }\end{array}$ & s.d. \\
\hline No Illness & $\ldots 4$ & $7 \cdot 03 \pm$ & $\cdot 12$ & 101 & $6 \cdot 55 \pm$ & $\cdot 10$ \\
$\begin{array}{l}\text { Cold or Slight Illness } \\
\text { Longer or More } \\
\text { Serious Illness }\end{array}$ & 68 & $6 \cdot 55 \pm$ & $\cdot 14$ & 95 & $6 \cdot 38 \pm$ & $\cdot 10$ \\
& 33 & $6 \cdot 78 \pm$ & $\cdot 19$ & 19 & $6 \cdot 32 \pm$ & $\cdot 23$ \\
\hline
\end{tabular}

For children of low nutritional grade (Investigation 2) the results were as follows:

33 children were marked down clinically at the first and second assessments; of these 25 gained less than the average weight during the year. Out of 64 who were marked down at the first assessment but were normal at the second assessment, 32 still failed to make the average weight gain. In addition, 43 children were marked "normal" at the first examination and were down-graded at the second; of these, 35 failed to put on the average increase of weight. That is to say, the children initially down-graded gained less weight in the following year than did the normal children (unless they reached the normal standard, in which case they tended to gain as much weight as the normal group), whilst children whose clinical status went down from first to second assessment put on even less weight.

The Rotherham Group Study (Investigation 5) included three groups of children aged 7 to 15 years:

(1) Children suffering from general nutritional debility attending an open-air school (nutritional group);

(2) Their brothers and sisters not requiring special treatment (sibling group);

(3) A control group of similar ages attending ordinary day schools.

Weight and height gains over the 3 years of the study are shown in Table V (overleaf).

During the first year the nutritional group gained least weight (less than the average L.C.C. child of comparable age (Scott, 1955), and considerably less than the control group). After the first year the nutritional group gained more weight than their brothers and sisters, and their height gain was greater than for the controls and was also above L.C.C. standards.

Home conditions were least good for the nutritional group children and their siblings, but during school hours conditions were most favourable for the nutritional group, and least favourable for their siblings. The results are dealt with more fully in another publication (Gillett and Hammond, in the press). 
TABLE V

GAIN IN WEIGHT AND HEIGHT ACCORDING TO NUTRITIONAL CATEGORY

\begin{tabular}{|c|c|c|c|c|c|c|c|c|c|c|c|c|c|c|c|c|c|c|}
\hline \multirow{3}{*}{ Year .. } & \multirow{3}{*}{$\cdots$} & \multirow{3}{*}{$\cdots$} & \multirow{3}{*}{$\cdots$} & \multicolumn{5}{|c|}{$1951-2$} & \multicolumn{5}{|c|}{$1952-3$} & \multicolumn{5}{|c|}{$1953-4$} \\
\hline & & & & \multicolumn{3}{|c|}{ Weight (kg.) } & \multicolumn{2}{|c|}{ Height $(\mathrm{cm})}$. & \multicolumn{3}{|c|}{ Weight (kg.) } & \multicolumn{2}{|c|}{ Height $(\mathrm{cm})}$. & \multicolumn{3}{|c|}{ Weight (kg.) } & \multicolumn{2}{|c|}{ Height (cm.) } \\
\hline & & & & No. & Mean & s.d. & Mean & s.d. & No. & Mean & s.d. & Mean & s.d. & No. & Mean & s.d. & Mean & s.d. \\
\hline \multicolumn{3}{|c|}{$\begin{array}{l}\text { Nutritional Group . . } \\
\text { Sibling Group } \\
\text { Control Group } \\
\text { L.C.C. Mean Differences }\end{array}$} & $\begin{array}{l}\ldots \\
\cdots \\
\cdots\end{array}$ & $\begin{array}{r}154 \\
46 \\
95 \\
-\end{array}$ & $\begin{array}{l}2 \cdot 86 \\
3 \cdot 27 \\
3 \cdot 89 \\
3 \cdot 67\end{array}$ & $\begin{array}{l}1 \cdot 27 \\
1 \cdot 72 \\
1 \cdot 76 \\
-\end{array}$ & $\begin{array}{l}5 \cdot 4 \\
5 \cdot 2 \\
5 \cdot 8 \\
5 \cdot 4\end{array}$ & $\begin{array}{l}1 \cdot 4 \\
1 \cdot 8 \\
1 \cdot 5 \\
-\end{array}$ & $\begin{array}{l}85 \\
21 \\
46 \\
-\end{array}$ & $\begin{array}{l}3 \cdot 63 \\
2 \cdot 86 \\
4 \cdot 72 \\
3 \cdot 86\end{array}$ & $\begin{array}{l}1 \cdot 45 \\
1 \cdot 81 \\
2 \cdot 03 \\
-\end{array}$ & $\begin{array}{l}5 \cdot 9 \\
4 \cdot 5 \\
6 \cdot 1 \\
5 \cdot 3\end{array}$ & $\begin{array}{l}1 \cdot 8 \\
1 \cdot 6 \\
1 \cdot 9 \\
\end{array}$ & $\begin{array}{l}73 \\
13 \\
43 \\
-\end{array}$ & $\begin{array}{l}3 \cdot 99 \\
3 \cdot 93 \\
4 \cdot 90 \\
4 \cdot 00\end{array}$ & $\begin{array}{l}1 \cdot 59 \\
1 \cdot 81 \\
3 \cdot 13 \\
-\end{array}$ & $\begin{array}{l}5 \cdot 7 \\
5 \cdot 7 \\
5 \cdot 0 \\
5 \cdot 5\end{array}$ & $\begin{array}{l}2 \cdot 1 \\
1 \cdot 7 \\
1 \cdot 9 \\
-\end{array}$ \\
\hline
\end{tabular}

For groups, weight and height growth shows differences consistent with their nutritional grading, as we should expect; but, for individuals, growth variability is so high that growth can only be used as an indication of nutritional condition in conjunction with other criteria.

\section{Physical Type}

During infancy the growth of two physical types-leptosomes (long, thin), and eurysomes (short, broad)-was studied in relation to type constancy (Hammond, 1957). Leptosomes were found to gain more weight relative to height than eurysomes.

For schoolchildren (Investigation 2) two types consisting of leptosomes and pyknics (short, fat, rounded type) were differentiated on the basis of twenty physical measurements. The mean annual weight growth of these types is shown in Table VI*

* In all cases the types were first differentiated and subsequent growth compared. Studies in which past growth of physical types is compared involve a circular reasoning, since leptosome types, for example, are just those children whose weight is low relative to height and in whom therefore weight growth has necessarily not kept pace with height growth.

TABLE VI

ANNUAL WEIGHT AND HEIGHT GAIN ACCORDING TO PHYSICAL TYPE

\begin{tabular}{|c|c|c|c|c|c|c|c|c|c|c|c|c|}
\hline \multirow{4}{*}{$\begin{array}{l}\text { Gain } \ldots \\
\begin{array}{ll}\text { Sex } \ldots & \cdots \\
\text { Type } \ldots & \ldots \\
\text { Age }(y r s)\end{array} \\
\end{array}$} & \multicolumn{8}{|c|}{ Weight (kg.) } & \multicolumn{4}{|c|}{ Height (cm.) } \\
\hline & \multicolumn{4}{|c|}{ Boys } & \multicolumn{4}{|c|}{ Girls } & \multicolumn{2}{|c|}{ Boys } & \multicolumn{2}{|c|}{ Girls } \\
\hline & \multicolumn{2}{|c|}{ Leptosome } & \multicolumn{2}{|c|}{ Pyknic } & \multicolumn{2}{|c|}{ Leptosome } & \multicolumn{2}{|c|}{ Pyknic } & \multirow{2}{*}{$\frac{\text { Leptosome }}{(\mathrm{cm} .)}$} & \multirow{2}{*}{$\frac{\text { Pyknic }}{(\mathrm{cm} .)}$} & \multirow{2}{*}{$\frac{\text { Leptosome }}{(\mathrm{cm} .)}$} & \multirow{2}{*}{$\frac{\text { Pyknic }}{(\mathrm{cm} .)}$} \\
\hline & No. & kg. & No. & $\mathrm{kg}$. & No. & kg. & No. & $\mathrm{kg}$. & & & & \\
\hline Birth & 31 & $7 \cdot 47$ & 22 & $6 \cdot 31$ & 26 & $7 \cdot 09$ & 42 & $6 \cdot 52$ & $22 \cdot 60$ & $20 \cdot 62$ & $22 \cdot 14$ & $20 \cdot 88$ \\
\hline 1 & 22 & $2 \cdot 54$ & 21 & $2 \cdot 30$ & 24 & $2 \cdot 47$ & 17 & $2 \cdot 47$ & $13 \cdot 75$ & $13 \cdot 40$ & $13 \cdot 85$ & \\
\hline 2 & 9 & 1.97 & 8 & $1 \cdot 80$ & 20 & $2 \cdot 03$ & 5 & $(1 \cdot 90)$ & $8 \cdot 60$ & $8 \cdot 68$ & $8 \cdot 59$ & $(8 \cdot 82)$ \\
\hline 3 & 10 & $2 \cdot 09$ & 7 & $(1 \cdot 28)$ & 15 & $1 \cdot 78$ & 16 & $2 \cdot 12$ & $6 \cdot 84$ & $7 \cdot 00$ & $7 \cdot 42$ & $7 \cdot 59$ \\
\hline 4 & 11 & $1 \cdot 51$ & 13 & $1 \cdot 81$ & 12 & 1.99 & 13 & $2 \cdot 00$ & $6 \cdot 32$ & $6 \cdot 10$ & $6 \cdot 09$ & $6 \cdot 34$ \\
\hline 5 & 23 & $1 \cdot 77$ & 14 & 1.91 & 24 & $2 \cdot 17$ & 22 & $2 \cdot 15$ & $5 \cdot 94$ & $5 \cdot 70$ & $5 \cdot 93$ & $5 \cdot 28$ \\
\hline 6 & 21 & $2 \cdot 04$ & 16 & $1 \cdot 70$ & 27 & $2 \cdot 53$ & 28 & $2 \cdot 02$ & $5 \cdot 12$ & $5 \cdot 42$ & $5 \cdot 33$ & $4 \cdot 97$ \\
\hline 7 & 25 & $2 \cdot 38$ & 23 & $2 \cdot 11$ & 37 & $2 \cdot 47$ & 32 & $2 \cdot 45$ & $5 \cdot 30$ & $5 \cdot 79$ & $4 \cdot 63$ & $4 \cdot 56$ \\
\hline 8 & 41 & $2 \cdot 40$ & 38 & $2 \cdot 45$ & 50 & $2 \cdot 93$ & 46 & $2 \cdot 74$ & $4 \cdot 79$ & $4 \cdot 60$ & $4 \cdot 70$ & $4 \cdot 57$ \\
\hline 9 & 54 & $2 \cdot 52$ & 55 & $2 \cdot 65$ & 48 & $2 \cdot 64$ & 45 & $2 \cdot 80$ & $4 \cdot 93$ & $4: 29$ & $4 \cdot 68$ & $4 \cdot 42$ \\
\hline 10 & 48 & $2 \cdot 95$ & 51 & $2 \cdot 65$ & 42 & $3 \cdot 53$ & 42 & $3 \cdot 94$ & $5 \cdot 84$ & $4 \cdot 13$ & $4 \cdot 64$ & $5 \cdot 08$ \\
\hline 11 & 52 & $3 \cdot 08$ & 46 & $3 \cdot 42$ & 52 & $4 \cdot 65$ & 29 & $4 \cdot 08$ & $4 \cdot 25$ & $4 \cdot 62$ & $5 \cdot 61$ & $4 \cdot 83$ \\
\hline 12 & 26 & $5 \cdot 47$ & 23 & $4 \cdot 30$ & 41 & $5 \cdot 28$ & 39 & $4 \cdot 42$ & 5.99 & $5 \cdot 28$ & $5 \cdot 90$ & $4 \cdot 77$ \\
\hline 13 & 15 & $6 \cdot 26$ & 22 & 4.61 & 41 & $4 \cdot 59$ & 29 & $2 \cdot 90$ & 6.05 & $5 \cdot 25$ & $4 \cdot 27$ & $2 \cdot 80$ \\
\hline 14 & 28 & $6 \cdot 25$ & 20 & $4 \cdot 89$ & 23 & $3 \cdot 26$ & 26 & $2 \cdot 44$ & $5 \cdot 20$ & $5 \cdot 40$ & $2 \cdot 39$ & $1 \cdot 38$ \\
\hline 15 & 23 & 4.90 & 28 & $4 \cdot 77$ & 21 & $3 \cdot 16$ & 22 & $1 \cdot 36$ & $3 \cdot 52$ & $4 \cdot 87$ & $1 \cdot 28$ & $1 \cdot 30$ \\
\hline 16 & 18 & $2 \cdot 76$ & 26 & $3 \cdot 19$ & 20 & $5 \cdot 31$ & 10 & 0.00 & $2 \cdot 19$ & 3.05 & 0.68 & 0.06 \\
\hline 17 & 16 & $2 \cdot 67$ & 7 & $(3 \cdot 07)$ & 一 & - & - & - & 0.90 & $2 \cdot 66$ & - & - \\
\hline
\end{tabular}



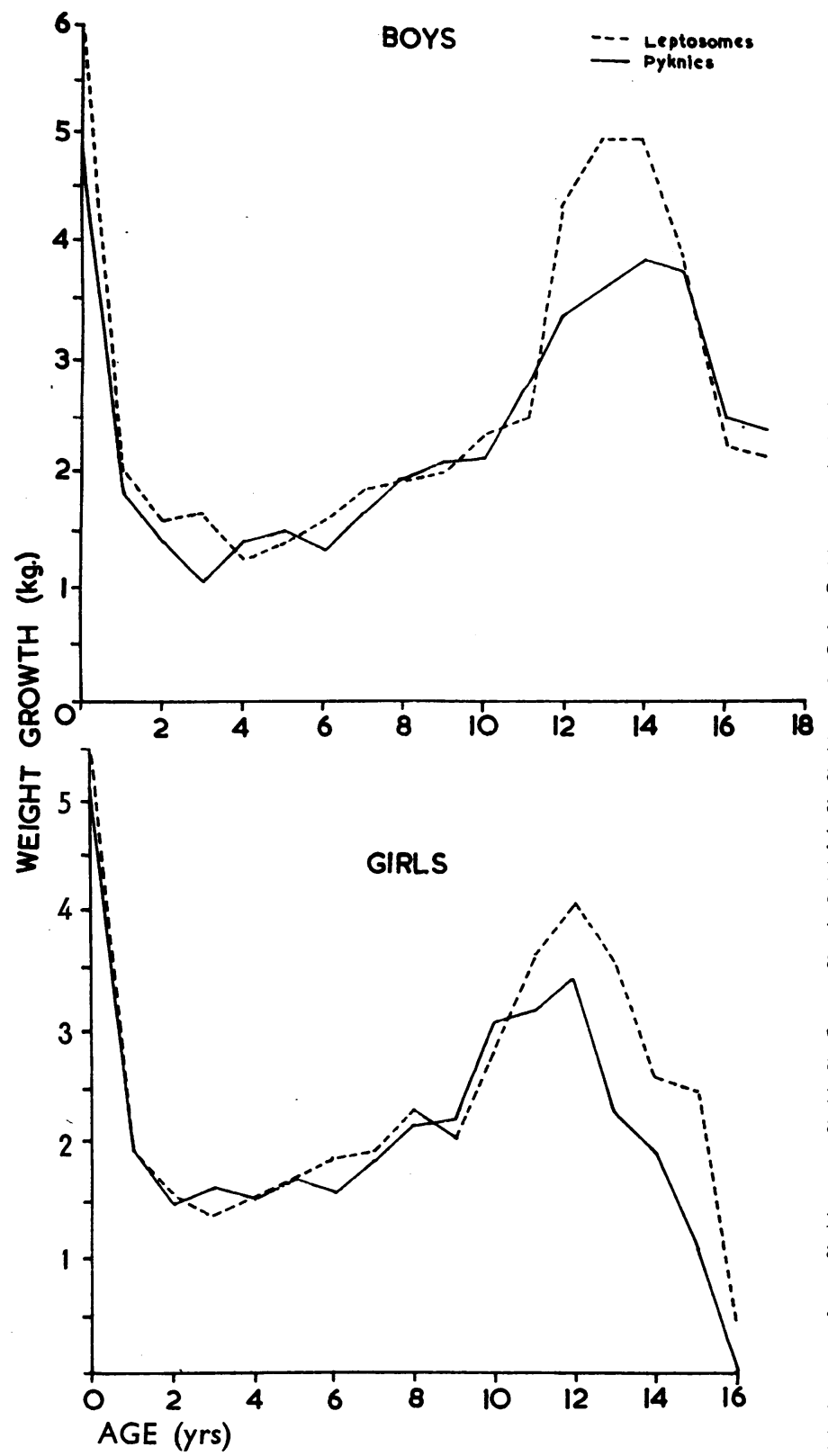

FIG. 3.-Weight growth in boys and girls of two physical types.

showing the leptosome habitus), and then proceed to "fill out" and gain more in weight. This is confirmed by the height increases, which are greater for the leptosomes between age 8 and age 13 .

\section{Stage of Development and Growth}

At puberty, mature children are considerably bigger than immature children of the same age (Ellis, 1946; Berry and Cowin, 1954; Scott, 1955 (boys); Wilson and Sutherland, 1950 (girls). Early maturers are also bigger from an early age (Ellis). Hammond (1955) has shown that the greater weight of children of higher social groups could be accounted for if we assumed that they matured earlier, and if we attributed to them the growth rates appropriate to their stage of development rather than to their chronological age. The cumulative effect of the greater growth due to accelerated maturity was seen to be sufficient to account for the group differences in attained weight. A similar analysis of yearly growth during the period of puberty in terms of the numbers of children at the different maturity levels and their appropriate growth has been made as follows:

The peak velocity of growth, based on children whose puberty stage is known and also on the data of Tanner (1956) may be estimated at about $7 \mathrm{~kg}$. per year, and the peak occurs at 14 years (s.d. 1 year). The whole spurt lasts for about 3 years, and we can accept peak growth for the years adjacent to the peak point as $7 \mathrm{~kg}$. per year, and for the outer years over which effects of the spurt are felt as $4.5 \mathrm{~kg}$., giving a growth pattern of $4 \cdot 5$, $7,7,4 \cdot 5 \mathrm{~kg}$.

The numbers of children whose peak occurs within given ages (calculated by probit analysis assuming a normal distribution) will then be as follows:

and in Fig. 3. The two physical types show little difference in the rate of growth from age 5 to $11+$ (boys) and from age 5 to $10+$ (girls), but from then onwards the leptosomes of both sexes show a somewhat greater spurt. This may be due to the fact that some children tend to increase their height without increasing comparably in weight before puberty (thus

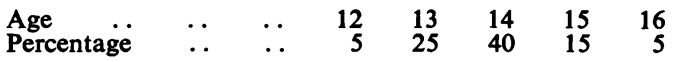

In a 14-year-old group, therefore, 40 per cent. are at their stage of maximum spurt, 25 per cent. have had their peak growth 1 year earlier, and 5 per cent. have had it 2 years earlier, whilst 25 per cent. are at a stage 1 year before their period of maximum growth, 
and 5 per cent: are 2 years before it. Similarly, in a 15-year-old group, 25 per cent. are at the peak of growth, 40 per cent. are 1 year past it, and so on. If we apply the assumed pattern of the spurt as above, with $2.7 \mathrm{~kg}$. per year as the prepubertal and $2 \cdot 2 \mathrm{~kg}$. as the post-pubertal level of growth, we obtain the following expected figures for the average growth for boys:

\begin{tabular}{c|c|c}
\hline \multirow{2}{*}{ Age (yrs) } & \multicolumn{2}{|c}{ Weight Gain (kg.) } \\
\cline { 2 - 3 } & Calculated & Actual \\
\hline $10+$ & $2 \cdot 8$ & $3 \cdot 0$ \\
$11+$ & $3 \cdot 4$ & $3 \cdot 5$ \\
$12+$ & $4 \cdot 7$ & $4 \cdot 6$ \\
$13+$ & $5 \cdot 9$ & $5 \cdot 4$ \\
$14+$ & $5 \cdot 9$ & $5 \cdot 8$ \\
$15+$ & $4 \cdot 5$ & $4 \cdot 6$ \\
$16+$ & $3 \cdot 1$ & $3 \cdot 1$ \\
\hline
\end{tabular}

The calculated figures agree fairly closely with the actual weight gains, the highest gains (for ages 13 and 14) being somewhat above those in Table I.

\section{Attained Measurements and Weight Gain}

The attained measurements will determine the level of growth to be expected, because certain conditions necessarily affect both, and because growth is to some degree proportional to the size of existing structures.

Correlations between attained measurements and one-year weight gains within social classes (Table VII) are positive at the earlier years and become negative around puberty.* At younger ages larger children gain more weight than smaller children simply because this is a continuation of the process that has made them larger. Just before puberty the larger children also have their growth spurts earlier. Over the next years, the growth of children who have had their spurt and are therefore bigger is reduced, whereas those who are small through not having reached their spurt grow more when they do begin it. Similarly, in late adolescence, the smaller children continue growth longer in order to reach their mature condition-for early puberty and early attainment of adult size both occur in favoured groups in whom the early development is greater.

These correlations are of little practical use in predicting growth. Even the multiple correlations between combinations of measurements and weight gain reach only $0 \cdot 40$.

When growth over a period of 2 years is correlated with the original measurements (Table.VIII, opposite), slightly higher figures are obtained, reaching 0.54 in the case of Weight and Hip Girth in the boys aged 8 to 11 years.

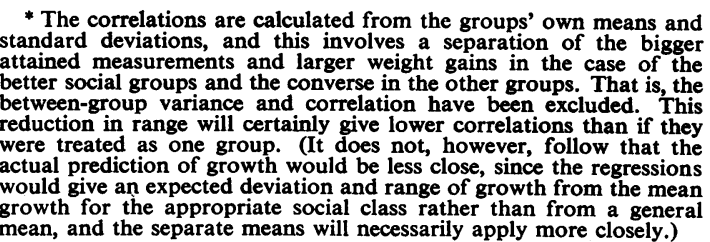

TABLE VII

CORRELATIONS BETWEEN BODY MEASUREMENTS AND ONE-YEAR WEIGHT GAIN DURING DIFFERENT GROWTH PHASES BY SEX

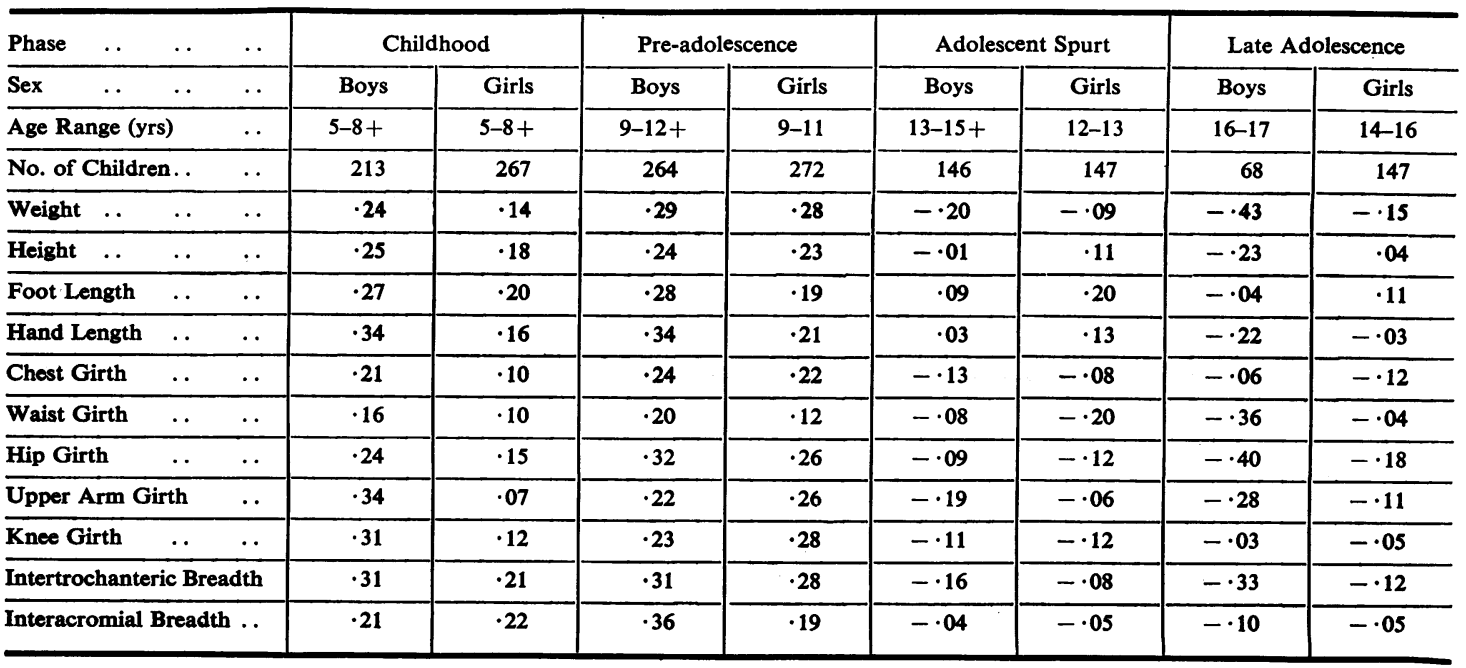


TABLE VIII

CORRELATIONS BETWEEN CERTAIN BODY MEASUREMENTS, AND WEIGHT GAIN OVER 2 YEARS, BY SOCIAL GROUP AND SEX

\begin{tabular}{|c|c|c|c|c|c|c|c|}
\hline \multirow{3}{*}{ Measurement } & \multirow{3}{*}{$\begin{array}{l}\text { Social } \\
\text { Group }\end{array}$} & \multicolumn{6}{|c|}{ Age (yrs) } \\
\hline & & \multicolumn{2}{|c|}{$5-7+$} & \multicolumn{2}{|c|}{$8-11+$} & \multicolumn{2}{|c|}{$12-15+$} \\
\hline & & Boys & Girls & Boys & Girls & Boys & Girls \\
\hline Weight & $\begin{array}{l}\mathbf{A} \\
\mathbf{B}\end{array}$ & $\overline{.44}$ & $\begin{array}{l}.23 \\
\cdot 40\end{array}$ & $\begin{array}{l}.40 \\
.54\end{array}$ & $\begin{array}{l}\cdot 15 \\
\cdot 40\end{array}$ & $\begin{array}{c}-\cdot 37 \\
-\end{array}$ & $\cdot 25$ \\
\hline Height & $\begin{array}{l}\mathbf{A} \\
\mathbf{B}\end{array}$ & $\overline{3} 7$ & $\begin{array}{l}.19 \\
.44\end{array}$ & $\begin{array}{l}.32 \\
.47\end{array}$ & $\begin{array}{l}\cdot 29 \\
\cdot 24\end{array}$ & -14 & $\stackrel{-37}{-}$ \\
\hline Foot Length & $\begin{array}{l}\mathbf{A} \\
\mathbf{B}\end{array}$ & $\overline{\cdot 27}$ & $\begin{array}{l}\cdot 06 \\
\cdot 31\end{array}$ & $\begin{array}{l}\cdot 34 \\
\cdot 38\end{array}$ & $\begin{array}{l}\cdot 33 \\
\cdot 27\end{array}$ & -04 & -37 \\
\hline Hip Girth .. & $\begin{array}{l}\mathbf{A} \\
\mathbf{B}\end{array}$ & $\overline{\cdot 34}$ & $\begin{array}{l}.26 \\
\cdot 43\end{array}$ & $\begin{array}{l}.31 \\
.46\end{array}$ & $\begin{array}{l}\cdot 15 \\
.40\end{array}$ & -26 & $\stackrel{\cdot 30}{-}$ \\
\hline Knee Girth. . & $\begin{array}{l}\mathbf{A} \\
\mathbf{B}\end{array}$ & $\overline{-38}$ & $\begin{array}{l}\cdot 18 \\
\cdot 27\end{array}$ & $\begin{array}{l}\cdot 37 \\
\cdot 46\end{array}$ & $\begin{array}{l}.15 \\
\cdot 50\end{array}$ & -12 & -31 \\
\hline $\begin{array}{l}\text { Inter- } \\
\text { trochanteric } \\
\text { Breadth } \quad \cdots\end{array}$ & $\begin{array}{l}\mathbf{A} \\
\mathbf{B}\end{array}$ & $\overline{.36}$ & $\begin{array}{l}.25 \\
.46\end{array}$ & $\begin{array}{l}\cdot 35 \\
\cdot 54\end{array}$ & $\begin{array}{l}\cdot 15 \\
\cdot 33\end{array}$ & $-\cdot 25$ & $\frac{\cdot 31}{-}$ \\
\hline $\begin{array}{c}\text { Interacromial } \\
\text { Breadth }\end{array}$ & $\begin{array}{l}\mathbf{A} \\
\mathbf{B}\end{array}$ & $\overline{.35}$ & $\begin{array}{l}\cdot 20 \\
\cdot 35\end{array}$ & $\begin{array}{l}.33 \\
\cdot 40\end{array}$ & $\begin{array}{l}\cdot 16 \\
\cdot 25\end{array}$ & $\begin{array}{c}-17 \\
-\end{array}$ & -36 \\
\hline $\begin{array}{l}\text { No. of } \\
\text { Children .. }\end{array}$ & $\begin{array}{l}\mathbf{A} \\
\mathbf{B}\end{array}$ & $\overline{98}$ & $\begin{array}{r}36 \\
104\end{array}$ & $\begin{array}{l}121 \\
112\end{array}$ & $\begin{array}{l}144 \\
141\end{array}$ & 134 & 129 \\
\hline
\end{tabular}

Note: Correlations are within year figures combined over the age groups shown; e.g. age group 12-15+ indicates growth from ages $12-14,13-15,14-16$, and 15-17 years.

These correlations are still of limited use in reducing the range of expected variation in growth, and the highest multiple correlations are only a little over $0 \cdot 6$.

There is little difference in predictability of growth for boys and girls, and also little difference from age 5 until the pubertal spurt begins. Throughout the age range, Social Group B (the poorer group) has higher correlations than the Social Group A, possibly because in this group some children are very small, on account of a depressed rate of growth in the past, and their low rate continues.

\section{Growth Consistency}

An Interpretation of the Variability of Growth over Short and Long Periods

The consistency in growth over different intervals can be determined by correlating the growth increases from one period to another; but where this may not be possible, as, for example, when the same children are not measured over the whole period, it can be inferred from the relations between growth variability during short and long periods.

The standard deviations of growth over 2 years or longer will equal the sum of those of successive yearly increases if the correlation between the separate years' growth is perfect. With zero correlation the variance will be additive and therefore the standard deviations will be equal to the square root of the sum of the variances. Negative correlation will result in a reduction of the overall variance compared with either of the yearly values.*

In the group of infants measured annually (Hammond, 1955), it was found that the sums of the variances of the yearly growth equalled the variances of the corresponding periods from birth to 5 years, indicating almost zero correlation between growth in any year compared with the next. Similarly the variances of the separate year's growth calculated from Table I are less than the variances of the 3-year growth up to age 12 and slightly greater from age 13 to age 15. These results show that growth from year to year has a low positive correlation up to age 12. After this, until age 15, negative correlations occur, since children tend to grow rapidly in one year and less rapidly in another, reflecting the growth trough before the pubertal spurt or different stages of the spurt.

The correlations between annual weight and height gain in non-adjacent periods for the data of Low (1952) and Hammond (1953) were as follows:

\begin{tabular}{|c|c|c|c|c|c|c|}
\hline Gain & Sex & $\begin{array}{l}\text { No. of } \\
\text { Child- } \\
\text { ren }\end{array}$ & $\begin{array}{c}\text { Birth } \\
1 \text { yr } \\
\text { with } \\
2-3 \text { yrs } \\
r\end{array}$ & $\begin{array}{c}1-2 \text { yrs } \\
\text { with } \\
3-4 \text { yrs } \\
r\end{array}$ & $\begin{array}{c}2-3 \text { yrs } \\
\text { with } \\
4-5 \text { yrs } \\
r\end{array}$ & $\begin{array}{c}8-9 \text { yrs } \\
\text { with } \\
10-11 \text { yrs } \\
r\end{array}$ \\
\hline \multirow{2}{*}{ Weight } & Boys & $(66)$ & -0.29 & -0.53 & -0.04 & (100) 0.118 \\
\hline & Girls & $(60)$ & -0.33 & -0.09 & -0.14 & (67) 0.355 \\
\hline \multirow{2}{*}{ Height } & Boys & (66) & $-0 \cdot 18$ & +0.01 & -0.16 & - \\
\hline & Girls & (60) & 0.10 & 0.04 & 0.06 & - \\
\hline
\end{tabular}

The author's own data gave correlations between growth from birth to 2 years and growth from 3 to 5 years, ranging from 0.08 to 0.26 for a number of different physical measurements.

The correlations are so low as to suggest that growth over different periods, even within the same stages of growth, is affected by conditions which vary from one moment to the next. Other body measurements show much the same unpredictability.

The correlations between growth from birth to 1 year and birth to 5 years were:

$$
\begin{aligned}
& \text { Weight : } \text { boys } 0.47 \\
& \text { girls } 0.59 \\
& \text { Height: } \text { boys } 0.63 \\
& \text { girls } 0.66
\end{aligned}
$$

* The relation is as follows:

Variance of Combined Growth $=V s_{1}+V s_{2}+2 r \sqrt{V} s_{1} V s_{2}$ where $V s_{1}$ and $V s_{2}$ are variances of separate annual growth increases, and $r$ is the correlation between them. 


\section{Discussion}

Growth velocity-to use Tanner's term-has in the past been treated less adequately than attained size. This, of course, is because studies of growth require at least two measurements and a time interval and are therefore more difficult to carry out.

After the fourth year of life the variability of annual weight increase is so high that any measureable increase, however small, lies within the "statistically normal" range ( \pm 2 s.d.). Annual increase in height is much less variable and failure to gain in any year is rare (as rare as failure to gain weight over 3 years). The effect of age on growth rates is much less than its effect on attained size (which represents the cumulative result of growth from conception). The fairly constant level of growth over the period from age 5 to age 8 or 9 permits the comparison of groups of children within this range, even when age means or distributions are not exactly the same. The growth pattern for the years preceding puberty is not distinct enough to enable us to estimate from it the maturity level of the individual, or to say when his spurt is likely to occur. There is, however, some evidence of a slight trough in growth just before the pubertal spurt begins.

The variance of growth can be used to indicate whether differences in measurements between individuals or groups could have arisen within a particular period. If limiting conditions of growth over any period (e.g. mean \pm 2 s.d.) are applied to the differences between the attained measurements, it is possible to say whether the difference between individuals or groups could be accounted for by growth during the period tested. And the extent to which these ranges overlap will indicate the likelihood that the difference has arisen during the given period. Conversely, it is possible to show how great the growth must have been during any period for present differences to have arisen within that period.

It would be expected that the whole course of past growth from birth would give a very strong indication of the growth during subsequent years, yet correlations between attained measurements and later growth are surprisingly low. Tanner, Healy, Lockhart, MacKenzie, and Whitehouse (1956) found that the prediction of adults' measurements from measurements made during infancy was little improved by taking into account the course of growth from birth. The correlations obtained could be attributed in large part to the inclusion of the earlier measurements in the later ones (nearly half the total growth in stature from birth having already occurred by age 5). Growth from birth to 5 years can only be predicted from the first year's growth to the extent of its contribution to the common variance, while growth during the first 5 years has a negligible predictive value for subsequent growth. This is confirmed by the fact that correlations between the gains in adjacent years or within a narrow age range are less than the correlations between the first year's growth and the growth from birth to 5 years (where overlap occurs).

Growth during one year is, of course, only one possible interval which might be considered. Just as it reflects conditions acting over a shorter time than attained size, so growth during still shorter intervals will further localize conditions. So far, the main use for these shorter intervals has been in studying growth in the first year of life and seasonal fluctuations. Half-yearly or even quarterly growth can also be useful in the adolescent period.

\section{SUMMARY}

The means and standard deviations of weight and height growth are given for periods of 1,2 , and 3 years from birth to 18 years. The yearly growth is also differentiated in terms of two social groups, two physical types, and different nutritional classes.

Growth is greatest during the first year both in absolute amounts and relative to its variability. At adolescence, when growth is almost as great, its variability is much higher, largely because of the different time of onset of the spurt. For this reason different standards should be used for pre- and post-pubescent children apart from age.

Children in higher social groups and those of better nutritional status grow more, as we should expect, but in the former case the excess growth is mainly in height, and in the latter it is mainly in weight. Leptosome and pachysome types (eurysomes or pyknics) differ little in weight growth up to age 12 , but after this age children whose height growth has tended to precede weight growth and who are therefore more leptosome tend to gain more weight.

The growth over 2- and 3-year intervals is such as to suggest that growth in each individual year is uncorrelated.

Correlations between attained measurements and weight gain (within the same social class) are very low, rarely exceeding 0.4 for 1 year's growth and 0.5 for 2 years' growth. At puberty they become negative.

Correlations between growth at one period and another are mostly around zero and they are again negative at puberty.

Differences in weight gain between groups at pubertal ages can be estimated from the numbers at 
the different stages of puberty, assuming a definite spurt pattern.

By relating differences in measurements to the variability of growth it is possible to separate influences of short or long duration.

I wish to thank Dr. E. R. Bransby of the Ministry of Health who initiated these studies, the National Physical Laboratory for machine analysis of much of the data, and Mr. T. O'Hara, Ministry of Health, for his helpful suggestions at the later stages.

\section{REFERENCES}

Acheson, R. M., and Hewitt, D. (1954). Hum. Biol. 26, 342.

Berry, W. T. C., and Cowin, P. J. (1954). Brit. med. J., 1, 847.
Clements, E. M. B., and Pickett, K. (1955). "Anthropometric Considerations of Children's Clothing." Dep. of Anatomy, Univ. Birmingham.

- (1956). "Anthropometric Considerations of School Chairs and Tables". Dep. of Anatomy, Univ. Birmingham.

Ellis, R. W. B. (1946). Arch. Dis. Childh., 21, 181.

Gillett, J. G., and Hammond, W. H. In the Press.

Hammond, W. H. (1953a). Hum. Biol., 25, 65.

(1953b). British Journal of Preventive and Social Medicine, 7, 231. (1955). Ibid., 9, 152.

(1957). Hum. Biol., 29, 40.

Low, A. (1952). "Growth of Children". University of Aberdeen.

Provis, H. S., and Ellis, R. W. B. (1955). Arch. Dis. Childh., 30, 328.

Scott, J. A. (1955). "Report on the Heights and Weights of School Pupils in the County of London in 1954". L.C.C. Report, No. 3885.

Tanner, J. M. (1955). “Growth at Adolescence”. Blackwell, Oxford. Healy, M. J. R., Lockhart, R. D., MacKenzie, J. D., and Whitehouse, R. H. (1956). Arch. Dis. Childh., 31, 372.

Thomson, J. (1956). British Journal of Preventive and Social Medicine, $10,128$.

Wilson, D. C., and Sutherland, I. (1950). Brit. med. J., 2, 863. 